\title{
«Translationale Medizin» am SAQM-Symposium
}

\section{Roxane Kübler ${ }^{a}$, Esther Kraft ${ }^{b}$, Christoph Bosshard ${ }^{c}$}

a MA, Operative Leiterin der Schweizerischen Akademie für Qualität in der Medizin SAQM der FMH; 'b lic. rer. oec., Leiterin Abteilung Daten, Demographie und Qualität DDQ der FMH; ${ }^{c}$ Dr. med., Vizepräsident der FMH, Departementsverantwortlicher Daten, Demographie und Qualität

Am 17. April 2018 zeichnet die SAQM im Rahmen eines Symposiums praxiserprobte Qualitätsprojekte des Schweizer Gesundheitswesens mit ihrem neuen Qualitätspreis «Innovation Qualité» aus. Nicht nur der Preis ist innovativ, sondern auch das Veranstaltungsthema, das sich der «Translationalen Medizin» widmet. Seien Sie dabei und melden Sie sich für das Symposium an!

Was brauchen Patienten für ihre Gesundheit? Und was brauchen Gesundheitsfachleute, um ihre Arbeit zu verbessern? Diesen und weiteren Fragen geht die Schweizerische Akademie für Qualität in der Medizin SAQM an ihrem ersten Symposium nach und verleiht in diesem Rahmen auch den SAQM-Qualitätspreis «Innovation Qualité» an Qualitätspioniere im Gesundheitswesen. Die Veranstaltung findet am Dienstagnachmittag, 17. April 2018, in Bern statt. Das Symposium ist interprofessionell ausgerichtet und wendet sich an qualitätsinteressierte Ärztinnen und Ärzte aller Fachrichtungen, an Qualitätsinteressierte weiterer medizinischer Berufsgruppen, an die Öffentlichkeit, an Pa- tientinnen und Patienten sowie an Politik und Medien. Sie sind herzlich willkommen!

\section{Innovationen aus Labor, Wissenschaft und vom Krankenbett}

Das Symposium der Innovation Qualité widmet sich mit einem Gastreferat dem Thema der «Translationalen Medizin». Die European Society for Translational Medicine (EUSTM) definiert Translationale Medizin als «ein[en] interdisziplinäre[n] Zweig der Biomedizin, der durch drei Hauptsäulen gestützt wird: die Forschung im Labor, die Behandlung am Krankenbett und die

\section{SFMH SAOM \} $F \mathrm{MH}_{\mathrm{ASQM}}$}

\section{Jetzt Projekt einreichen - bis 08.12.2017}

Zukunftsweisende Qualitätsarbeit im Gesundheitswesen gehört aufs Podest: Gewinnen Sie die Innovation Qualité, den neuen Preis für Qualität in der Medizin! Melden Sie Ihr Projekt an auf www.innovationqualite.ch. 
Gemeinschaft der Wissenschaftler. Das Ziel der Translationalen Medizin ist es, verschiedene Disziplinen, Ressourcen, Expertisen und technisches Know-how aus dem Labor, vom Krankenbett und aus der Wissenschaft bzw. von Wissenschaftlerinnen und Wissenschaftlern zusammenzubringen, um eine Weiterentwicklung und Verbesserung der Prävention, Diagnose und Therapie zu fördern.» [1, 2] Neue Erkenntnisse aus Forschung und Entwicklung sollen also nicht dort verhaftet bleiben. Vielmehr sollen aus diesen Erkenntnissen innovative Produkte und Instrumente entstehen,

Korrespondenz:

FMH Verbindung der

Schweizer Ärztinnen

und Ärzte

Abteilung Daten, Demo-

graphie und Qualität

Elfenstrasse 18

Postfach 300

CH-3000 Bern 15

roxane.kuebler[at]fmh.ch

Tel. 0313591111 die den Markt und damit auch die Patienten erreichen.

\section{Translationale Medizin in Theorie und Praxis}

Das Swiss Institute for Translational and Entrepreneurial Medicine (sitem-insel AG) bezweckt den Aufbau und Betrieb eines nationalen Zentrums für Trans- lationale Medizin und Unternehmertum in Bern [3]. Prof. Dr. med., em. Felix Frey, Geschäftsführer der siteminsel AG, wird am Symposium Chancen und Nutzen für die Patientin, den Patienten vorstellen.

\section{Anmeldung}

Sie können sich ab sofort für das SAQM-Symposium anmelden: www.innovationqualite.ch $\rightarrow$ Preisverleihung am (SAQM-)Symposium. Wir freuen uns auf Sie!

\section{Literatur}

1 Randall J. Cohrs, Tyler Martin, Parviz Ghahramani, Luc Bidaut Paul J. Higgins und Aamir Shahzad: Translational Medicine definition by the European Society for Translational Medicine, New Horizons in Translational Medicine, März 2015, Band 2 (3), S. 86-88. http://www.newhorizonsintranslationalmedicine.com/ article/S2307-5023(14)00078-2/pdf [4.10.2017].

2 https://de.wikipedia.org/wiki/Translationale_Medizin [4.10.2017].

3 vgl.http://www.sitem-insel.ch [4.10.2017]. 